\title{
Unified Composite Distribution with Applications to Double Shadowed $\kappa-\mu$ Fading Channels
}

\author{
Hussien Al-Hmood, Member, IEEE, and H. S. Al-Raweshidy, Senior Member, IEEE
}

\begin{abstract}
In this paper, a mixture Gamma shadowed (MGS) model is proposed as a unified composite distribution via representing the shadowing impact by an inverse Nakagami- $m$. The exact expression and the asymptotic behaviour at high average signal-to-noise ratio (SNR) regime of the fundamental statistics of a MGS distribution are derived first. These statistics are then applied to analyze the performance of the wireless communication systems over double shadowed $\kappa$ - $\mu$ fading channels. In particular, the outage probability (OP), average bit error probability (ABEP), average channel capacity (ACC), effective capacity (EC) and average area under the receiver operating characteristics curve (AUC) of energy detection (ED) are provided. The numerical and simulation results as well as a comparison with previous exact works are presented to verify the validation of our analysis.
\end{abstract}

Index Terms-Mixture Gamma shadowed, double shadowed $\kappa-\mu$ fading, average bit error probability, capacity analysis.

\section{INTRODUCTION}

$\mathbf{T}$ HE wireless communications channel may undergo the effect of the multipath and shadowing fading simultaneously [1]. Accordingly, many works have been dedicated to analyze the performance of the communication systems over generalized fading channels, such as, $\kappa-\mu$ and $\eta-\mu$ [2]. These generalized conditions can provide close results to the practical measurements and approximately comprise all the classical fading distributions. Hence, the probability density function (PDF), cumulative distribution function (CDF), and moment generating function (MGF) of the composite $\eta-\mu / \mathrm{Gamma}$ fading models were derived in [3] with applications to the outage probability (OP), average bit error probability (ABEP) and average channel capacity (ACC). The PDF and CDF of composite $\kappa-\mu /$ Gamma fading were presented in [4]. The statistics of the $\kappa-\mu$ shadowed fading in which the dominant components are shadowed by a Nakagami- $m$ were given in [5]. The authors in [6] assumed that both $\kappa-\mu$ and $\eta-\mu$ fading models are shadowed by an inverse Gamma distribution.

Recently, several studies have been explained that the fading channels may subject to double shadowing impacts at the same time. For example, in [7], the statistical properties of the double shadowed Rician fading channel were derived. In [8],

Manuscript received November 00, 2020.

Copyright (c) 2015 IEEE. Personal use of this material is permitted. However, permission to use this material for any other purposes must be obtained from the IEEE by sending a request to pubs-permissions@ieee.org.

Hussien Al-Hmood is with the Department of Electrical and Electronics Engineering, College of Engineering, University of Thi-Qar, Iraq, e-mail: Hussien.Al-Hmood@utq.edu.iq.

H. S. Al-Raweshidy is with the Department of Electronic and Computer Engineering, College of Engineering, Design and Physical Sciences, Brunel University London, UK, e-mail: Hamed.Al-Raweshidy@brunel.ac.uk. the performance of unmanned aerial vehicle (UAV)-enabled wireless communication systems over composite cascaded Nakagami- $m$ /double inverse Gamma fading channels was analyzed. Different scenarios of the double shadowed $\kappa$ - $\mu$ fading using Nakagami- $m$ and inverse Nakagami- $m$ distributions were investigated in [9]. However, the derived statistics of [9] include either the infinite series or mathematically intractable function, such as, Kampe de Feriet function [9, eq. (26) that is not available in the popular software packages.

More recently, a mixture Gamma (MG) distribution has been widely used in the literature [10]. This distribution can approximate with high accuracy most of the composite generalized/Gamma fading channels, e.g., $\kappa-\mu /$ Gamma and $\eta$ $\mu /$ Gamma [11], $\alpha-\eta-\mu /$ Gamma [12], generalized- $K\left(K_{G}\right)$ [13], and $\alpha-\eta-\lambda-\mu / \mathrm{Gamma}[14]$.

Based on the above observations, in this paper, a MG shadowed (MGS) distribution is proposed as a unified composite model where the shadowing is represented by an inverse Nakagami- $m$. To this end, the basic statistics are mathematically simple and tractable. Consequently, the derived performance metrics over double shadowed fading channels have low complexity in comparison with the exact expressions.

Our main contributions are summarized as follows:

- Providing the exact expression and asymptotic behaviour at high average signal-to-noise ratio (SNR) values of the statistical characterization of MGS fading model.

- Capitalizing on the above, we analyze the performance of the wireless communication systems over double shadowed $\kappa-\mu$ fading channel in which the shadowing of the dominant components is modelled by a Nakagami- $m$ distribution. Hence, the parameters of a MG distribution of composite $\kappa-\mu /$ Nakagami- $m$ fading are derived.

- To this effect, novel unified mathematically tractable closed-form expressions for the ABEP, ACC, effective capacity (EC), and average area under the receiver operating characteristics (AUC) curve of the energy detection (ED) based spectrum sensing are obtained.

\section{Statistical Properties of a MGS Distribution}

The PDF of a MG distribution is given by [10, eq. (1)]

$$
f(x)=\sum_{j=1}^{K} \sigma_{j} x^{\beta_{j}-1} e^{-\zeta_{j} x}
$$

where $\sigma_{j}, \beta_{j}$, and $\zeta_{j}$ are the parameters of $j$ th Gamma component and $K$ that stands for the number of terms, can be evaluated via using the mean square error (MSE) method between the exact PDF and its MG representation [10]. 
If $\xi$ is an inverse Nakagami- $m$ random variable (RV) with $\mathbb{E}[\xi]=1$ where $\mathbb{E}[$.$] indicates the statistical expectation, its$ power normalized PDF is expressed as [9, eq. (50)]

$$
f_{\xi}(r)=\frac{\left(m_{s}-1\right)^{m_{s}}}{\Gamma\left(m_{s}\right) r^{m_{s}+1}} e^{-\frac{\left(m_{s}-1\right)}{r}} m_{s}>1
$$

where $m_{s}$ refers to the shadowing severity index and $\Gamma($.$) is$ the Gamma function [15, eq. (8.310.1)].

Let $\gamma \sim \operatorname{MGS}\left(\sigma_{j}, \beta_{j}, \zeta_{j}, m_{s}\right)$ for $j=1, \cdots, K$ is an MGSdistributed RV. Then, the PDF of $\gamma$ can be obtained by the product of MG and inverse Nakagami- $m$ RVs as [3, eq. (8)]

$$
f_{\gamma}(\gamma)=\int_{0}^{\infty} \frac{1}{z} f\left(\frac{\gamma}{z}\right) f_{\xi}(z) d z
$$

Substituting (1) and (2) into (3) and making use of [15, eq. (3.381.4)] with some mathematical manipulations, this yields

$$
f_{\gamma}(\gamma)=\left(m_{s}-1\right)^{m_{s}} \sum_{j=1}^{K} \frac{\sigma_{j}\left(m_{s}\right)_{\beta_{j}} \gamma^{\beta_{j}-1}}{\left(m_{s}-1+\zeta_{j} \gamma\right)^{\beta_{j}+m_{s}}}
$$

where $(.)_{n}$ is the Pochhammer symbol.

When $\zeta_{j} \rightarrow 0$ for all $j=1, \cdots, K$, the asymptotic of the PDF, $f_{\gamma}^{\infty}(\gamma)$, can be expressed as

$$
f_{\gamma}^{\infty}(\gamma) \simeq \sum_{j=1}^{K} \frac{\sigma_{j}\left(m_{s}\right)_{\beta_{j}} \gamma^{\beta_{j}-1}}{\left(m_{s}-1\right)^{\beta_{j}}}
$$

Inserting (4) in $F_{\gamma}(\gamma)=\int_{0}^{\gamma} f_{\gamma}(\gamma) d \gamma$ and recalling [15, eq. (3.194.1)], the CDF of a MGS distribution can be derived as

$$
F_{\gamma}(\gamma)=\sum_{j=1}^{K} \frac{\sigma_{j}\left(m_{s}\right)_{\beta_{j} 2} F_{1}\left(\beta_{j}+m_{s}, \beta_{j} ; \beta_{j}+1 ;-\frac{\zeta_{j}}{m_{s}-1} \gamma\right)}{\beta_{j}\left(\left(m_{s}-1\right) \gamma^{-1}\right)^{\beta_{j}}}
$$

where ${ }_{2} F_{1}(., . ; . ;$.$) is the Gauss hypergeometric function de-$ fined in [15, eq. (9.14.1)].

Using the fact that ${ }_{2} F_{1}(., . ; . ; 0) \simeq 1$ when $\zeta_{j} \rightarrow 0$ or plugging (5) in $F_{\gamma}(\gamma)=\int_{0}^{\gamma} f_{\gamma}(\gamma) d \gamma$, the asymptotic of the CDF, $F_{\gamma}^{\infty}(\gamma)$, can be evaluated as

$$
F_{\gamma}^{\infty}(\gamma) \simeq \sum_{j=1}^{K} \frac{\sigma_{j}\left(m_{s}\right)_{\beta_{j}} \gamma^{\beta_{j}}}{\beta_{j}\left(m_{s}-1\right)^{\beta_{j}}}
$$

Using the Laplace transform and invoking [16, eq. (07.33.07.0001.01)], the MGF of the MGS distribution can be obtained as

$$
\mathcal{M}_{\gamma}(s)=\sum_{j=1}^{K} \frac{\sigma_{j}\left(m_{s}\right)_{\beta_{j}} \Gamma\left(\beta_{j}\right) U\left(\beta_{j} ; 1-m_{s} ; \frac{m_{s}-1}{\zeta_{j}} s\right)}{\zeta_{j}^{\beta_{j}}}
$$

where $U(. ; . ;$.$) is the Tricomi confluent hypergeometric func-$ tion of the second kind defined in [15, eq. (9.211.4)].

The asymptotic of the MGF, $\mathcal{M}_{\gamma}^{\infty}(s)$, can be deduced after applying the Laplace transform for (5) and invoking [15, eq. (8.310.1)]. Thus, this yields

$$
\mathcal{M}_{\gamma}^{\infty}(s) \simeq \sum_{j=1}^{K} \frac{\sigma_{j}\left(m_{s}\right)_{\beta_{j}} \Gamma\left(\beta_{j}\right)}{\left[\left(m_{s}-1\right) s\right]^{\beta_{j}}}
$$

The $n$-th moment, $\boldsymbol{\mu}_{n}$, of the MGS distribution can be found by using (4) and [15, eq. (3.194.3)] as

$$
\boldsymbol{\mu}_{n}=\mathbb{E}\left[\gamma^{n}\right]=\sum_{j=1}^{K} \frac{\sigma_{j}\left(m_{s}\right)_{\beta_{j}} B\left(\beta_{j}+n, m_{s}-n\right)}{\zeta_{j}^{\beta_{j}+n}\left(m_{s}-1\right)^{-n}}
$$

where $B(.,$.$) is the Beta function [15, eq. (8.380.1)].$

It is worth mentioning that the MGS distribution can be used to model the $\kappa$ - $\mu$ /inverse Gamma fading [6] with $\sigma_{j}=$ $\frac{e^{-\mu \kappa} \mu^{\mu+2(j-1)} \kappa^{j-1}(1+\kappa)^{\mu+j-1}}{\Gamma(\mu+j-1) \Gamma(j) \bar{\gamma}^{\mu+j-1}}, \beta_{j}=\mu+j-1$, and $\zeta_{j}=\frac{\mu(1+\kappa)}{\bar{\gamma}}$. Additionally, the statistics of the Fisher Senedcor $\mathcal{F}$ fading [17] can be represented by (4)-(10) with $K=1, \sigma_{1}=\frac{m^{m}}{\Gamma(m) \bar{\gamma}^{m}}$, $\beta_{1}=m$, and $\zeta_{1}=\frac{m}{\bar{\gamma}}$.

\section{Double Shadowed $\kappa-\mu$ Fading Channels}

The received signal envelope, $R$, over double shadowed $\kappa-$ $\mu$ fading channel can be given as $[9$, eq. (22)]

$$
R^{2}=\xi^{2} \sum_{l=1}^{\mu}\left(X_{l}+\vartheta p_{l}\right)^{2}+\left(Y_{l}+\vartheta q_{l}\right)^{2}
$$

where the parameters of (11) are defined as follows:

i) $\mu$ is a real-valued extension related to the number of multipath clusters.

ii) $\xi$ and $\vartheta$ represent the $\mathrm{RVs}$ which are responsible for introducing the shadowing impacts that are modelled by inverse Nakagami- $m$ and Nakagami- $m$ distributions, respectively, with $\mathbb{E}\left[\xi^{2}\right]=\mathbb{E}\left[\vartheta^{2}\right]=1$. Physically, the effect of $\vartheta$ may occur when the signal power is shadowed by obstacles moving between the transmitter and receiver, such as, cars and/or people. Furthermore, the second impact, namely, $\xi$, may occur due to objects moving near to the transmitter and/or receiver [8], [9].

iii) $X_{l}$ and $Y_{l}$ are mutually independent Gaussian random processes with mean $\mathbb{E}\left[X_{l}\right]$ and $\mathbb{E}\left[Y_{l}\right]=0$ and variance $\mathbb{E}\left[X_{l}^{2}\right]=\mathbb{E}\left[Y_{l}^{2}\right]=\delta^{2}$.

iv) $p_{l}$ and $q_{l}$ are the mean values of the in-phase and quadrature phase components of the multipath cluster $l$. Hence, the power of the dominant component which is a complex RV expressed as $\vartheta p_{l}+i \vartheta q_{l}$, is $P_{l}=p_{l}^{2}+q_{l}^{2}$.

According to (11), the PDF of $R$ can be derived by averaging the PDF of the single shadowed $\kappa-\mu$ fading Type I model [9, eq. (2)] over (2). However, [9, eq. (2)] includes the confluent hypergeometric function ${ }_{1} F_{1}(. ; . ;$.$) [15, eq. (9.41.1)]$ that would lead to statistics in terms of either the infinite series or numerically complicated functions (please see [9, eqs. (25) and (26)]). Thus, to obtain simple closed-form expressions, the PDF of the induced shadowing of the dominant component is approximated by a MG distribution, whereas the multiplicative shadowing is represented by a MGS model.

The PDF of the received instantaneous SNR over single shadowed $\kappa-\mu$ Type I model is given by [9, eq. (3)]

$$
f(x)=\frac{\Omega^{\mu} m^{m} x^{\mu-1} e^{-\Omega x}}{\Gamma(\mu)(\mu \kappa+m)^{m}}{ }_{1} F_{1}\left(m ; \mu ; \frac{\mu \kappa \Omega}{\mu \kappa+m} x\right)
$$

where $\Omega=\frac{\mu(1+\kappa)}{\bar{\gamma}}, \bar{\gamma}$ is the average SNR, $\kappa$ is the ratio between the total powers of the dominant components $\left(d^{2}=\right.$ $\left.\sum_{l=1}^{\mu} P_{l}\right)$ and scattered waves $\left(2 \mu \delta^{2}\right)$, and $m$ is the shadowing severity index of the Nakagami- $m$. 
With the aid of [15, eq. (9.41.1)], (12) can be rewritten as $f(x)=\sum_{j=1}^{\infty} \frac{\Gamma(m+j-1) m^{m}(\kappa \mu)^{j-1} \Omega^{\mu+j-1} x^{\mu+j-2} e^{-\Omega x}}{\Gamma(m) \Gamma(\mu+j-1)(j-1) !(\mu \kappa+m)^{m+j-1}}$

The required accuracy for approximating (1) can be obtained by using $K$ terms for the series of (13). Hence, by matching (1) and (13), we have

$$
\begin{gathered}
\sigma_{j}=\frac{\Gamma(m+j-1) m^{m}(\kappa \mu)^{j-1} \Omega^{\mu+j-1}}{\Gamma(m) \Gamma(\mu+j-1)(j-1) !(\mu \kappa+m)^{m+j-1}} \\
\beta_{j}=\mu+j-1, \quad \zeta_{j}=\Omega
\end{gathered}
$$

\section{Performance Analysis using a MGS Model}

\section{A. Outage Probability}

The OP is defined as the probability of falling the values of the output SNR below a predefined threshold value $\varphi$.

The OP, $P_{o}$, can be computed by [1, eq. (1.4)]

$$
P_{o}=F_{\gamma}(\varphi)
$$

where $F_{\gamma}($.$) is provided in (6).$

The asymptotic of the OP, $P_{o}^{\infty}$, can be analyzed by (7), i.e., $P_{o}^{\infty}=F_{\gamma}^{\infty}(\varphi)$. Furthermore, the $P_{o}^{\infty}$ may be closely represented as $P_{o}^{\infty} \simeq\left(G_{c} \bar{\gamma}\right)^{-G_{d}}$ whereby $G_{c}$ denotes the coding gain and $G_{d}$ stands for the diversity order that demonstrates the increasing in the slope of the OP versus $\bar{\gamma}$.

For the double shadowed $\kappa-\mu$ fading and after plugging (14) in (7) and following a similar procedure of [18, eq. (14)], one obtains $G_{c}=\frac{\left(m_{s}-1\right)}{\mu(1+\kappa) \varphi}\left(\frac{\Gamma(\mu+1)(\mu \kappa+m)^{m}}{m^{m}\left(m_{s}\right)_{\mu}}\right)^{\frac{1}{\mu}}$ and $G_{d}=\mu$.

\section{B. Average Bit Error Probability}

The ABEP, $\bar{P}_{e}$, can be evaluated by [1, eq. (9.11)]

$$
\bar{P}_{e}=\frac{1}{\pi} \int_{0}^{\frac{\pi}{2}} \mathcal{M}_{\gamma}\left(\frac{\rho}{\sin ^{2} \phi}\right) d \phi
$$

where $\rho=0.5$ and $\rho=1$ for binary frequency shift keying (BFSK) and binary phase shift keying (BPSK), respectively.

Substituting (8) into (16) and utilizing the property [16, eq. (07.33.26.0004.01)], we have

$$
\bar{P}_{e}=\frac{1}{\pi \Gamma\left(m_{s}\right)} \sum_{j=1}^{K} \frac{\sigma_{j}}{\zeta_{j}^{\beta_{j}}} \int_{0}^{\frac{\pi}{2}} G_{1,2}^{2,1}\left[\frac{\left(m_{s}-1\right) \rho}{\zeta_{j} \sin ^{2} \phi} \mid \begin{array}{c}
1-\beta_{j} \\
0, m_{s}
\end{array}\right] d \phi
$$

where $G_{c, d}^{a, b}[$.$] is the Meijer's G$-function [15, eq. (9.301)].

Employing [16, eq. (07.34.02.0001.01)] and the change of variable $t=\sin ^{2} \phi$, (17) becomes

$$
\begin{gathered}
\bar{P}_{e}=\frac{1}{2 \pi \Gamma\left(m_{s}\right)} \sum_{j=1}^{K} \frac{\sigma_{j}}{\zeta_{j}^{\beta_{j}}} \int_{0}^{1} \frac{1}{2 \pi i \sqrt{(1-t) t}} \int_{\mathcal{L}} \Gamma(r) \Gamma\left(m_{s}+r\right) \\
\Gamma\left(\beta_{j}-r\right)\left(\frac{\left(m_{s}-1\right) \rho}{t \zeta_{j}}\right)^{-r} d r d t
\end{gathered}
$$

where $i=\sqrt{-1}$ and $\mathcal{L}$ is the suitable contour in the $r$-plane from $\varrho-i \infty$ to $\varrho+i \infty$ with $\varrho$ is a constant value.
Changing the order of the integrals of (18) and then utilizing [15, eq. (3.191.3)] for the linear integral, this yields

$$
\begin{gathered}
\bar{P}_{e}=\frac{1}{2 \pi \Gamma\left(m_{s}\right)} \sum_{j=1}^{K} \frac{\sigma_{j}}{\zeta_{j}^{\beta_{j}}} \frac{1}{2 \pi i} \int_{\mathcal{L}} \Gamma(r) \Gamma\left(m_{s}+r\right) \Gamma\left(\beta_{j}-r\right) \\
B(r+0.5,0.5)\left(\frac{\left(m_{s}-1\right) \rho}{\zeta_{j}}\right)^{-r} d r
\end{gathered}
$$

Recalling [15, eq. (8.384.1)/ eq. (8.338.2)] and using [18, eq. (07.34.02.0001.01)], (19) can be expressed as

$$
\bar{P}_{e}=\frac{1}{2 \sqrt{\pi} \Gamma\left(m_{s}\right)} \sum_{j=1}^{K} \frac{\sigma_{j} G_{2,2}^{2,1}\left[\frac{\left(m_{s}-1\right) \rho}{\zeta_{j}} \mid \begin{array}{l}
1-\beta_{j}, 1 \\
0, m_{s}, 0.5
\end{array}\right]}{\zeta_{j}^{\beta_{j}}}
$$

The asymptotic of the ABEP at high $\bar{\gamma}$ regime, $\bar{P}_{e}^{\infty}$, can be deduced after inserting (9) in (17) and using $t=\sin ^{2} \phi$ as

$$
\bar{P}_{e}^{\infty} \simeq \frac{1}{2 \pi} \sum_{j=1}^{K} \frac{\sigma_{j}\left(m_{s}\right)_{\beta_{j}} \Gamma\left(\beta_{j}\right)}{\left[\left(m_{s}-1\right) \rho\right]^{\beta_{j}}} \int_{0}^{1} \frac{t^{\beta_{j}}}{\sqrt{(1-t) t}} d t
$$

Invoking [15, eq. (3.191.3)], (21) can be evaluated as

$$
\bar{P}_{e}^{\infty} \simeq \frac{1}{2 \sqrt{\pi}} \sum_{j=1}^{K} \frac{\sigma_{j}\left(m_{s}\right)_{\beta_{j}} \Gamma\left(\beta_{j}+0.5\right)}{\beta_{j}\left[\left(m_{s}-1\right) \rho\right]^{\beta_{j}}}
$$

Substituting (14) into (22) and using the same steps of [18, eq. (14)], we have $G_{c}=\frac{\left(m_{s}-1\right) \rho}{\mu(1+\kappa)}\left(\frac{2 \sqrt{\pi} \Gamma(\mu+1)(\mu \kappa+m)^{m}}{m^{m}\left(m_{s}\right)_{\mu} \Gamma(\mu+0.5)}\right)^{\frac{1}{\mu}}$ and $G_{d}=\mu$ for the ABEP over double shadowed $\kappa-\mu$ fading.

\section{Average Channel Capacity}

The normalized ACC can be determined by [17, eq. (26)]

$$
\bar{C}=\frac{1}{\ln (2)} \int_{0}^{\infty} \ln (1+\gamma) f_{\gamma}(\gamma) d \gamma
$$

Plugging (4) in (23) and making use of the identity [16, eq. (01.04.26.0002.01)], we obtain

$\bar{C}=\frac{\left(m_{s}-1\right)^{m_{s}}}{\ln (2)} \sum_{j=1}^{K} \sigma_{j}\left(m_{s}\right)_{\beta_{j}} \int_{0}^{\infty} \frac{\gamma^{\beta_{j}-1} G_{2,2}^{1,2}\left[\begin{array}{l}\mid \\ 1,1 \\ 1,0\end{array}\right] d \gamma}{\left(m_{s}-1+\zeta_{j} \gamma\right)^{\beta_{j}+m_{s}}}$

Invoking [15, eq. (7.811.5)], (24) can be calculated as

$$
\bar{C}=\frac{1}{\ln (2) \Gamma\left(m_{s}\right)} \sum_{j=1}^{K} \frac{\sigma_{j}}{\zeta_{j}^{\beta_{j}}} G_{3,3}^{2,3}\left[\frac{m_{s}-1}{\zeta_{j}} \mid \begin{array}{c}
1-\beta_{j}, 1,1 \\
m_{s}, 1,0
\end{array}\right]
$$

The asymptotic of the ACC for $\bar{\gamma} \rightarrow \infty, \bar{C}^{\infty}$, can be evaluated via [17, eq. (28)]

$$
\left.\bar{C}^{\infty} \simeq \frac{1}{\ln (2)} \frac{\partial}{\partial n} \mathbb{E}\left[\gamma^{n}\right]\right|_{n=0}
$$

Substituting (10) into (26), computing the partial derivative, and setting $n=0, \bar{C}^{\infty}$ over MGS model is deduced as

$$
\bar{C}^{\infty} \simeq \sum_{j=1}^{K} \frac{\sigma_{j} \Gamma\left(\beta_{j}\right)\left[\ln \left(\frac{m_{s}-1}{\zeta_{j}}\right)+\psi\left(\beta_{j}\right)-\psi\left(m_{s}\right)\right]}{\ln (2) \zeta_{j}^{\beta_{j}}}
$$




$$
\overline{\mathcal{A}}=1-\sum_{l=0}^{u-1} \sum_{i=0}^{l}\left(\begin{array}{c}
l+u-1 \\
l-i
\end{array}\right) \frac{2^{-(l+i+u)}}{i !}\left(m_{s}-1\right)^{i} \sum_{j=1}^{K} \sigma_{j}\left(m_{s}\right)_{\beta_{j}} \Gamma\left(\beta_{j}+i\right) U\left(\beta_{j}+i ; i-m_{s}+1 ; \frac{m_{s}-1}{2 \zeta_{j}}\right)
$$

where $\psi($.$) is the Euler's digamma function [15, eq. (8.360.1)].$

\section{Effective Capacity}

In Shannon's theorem, the ACC has been measured under perfect quality of service (QoS). However, in the EC, the constraints of the QoS, such as, system delay, are taken into consideration [6]. The EC can be calculated by [12, eq. (4)]

$$
\mathcal{R}=-\frac{1}{A} \log _{2}\left\{\int_{0}^{\infty}(1+\gamma)^{-A} f_{\gamma}(\gamma) d \gamma\right\}
$$

where $A \triangleq \theta T B / \ln (2)$ with $\theta, T$, and $B$ denote the delay exponent, the time and the bandwidth of the channel.

Inserting (4) in (29) and employing [15, eq. (3.197.1)], the EC over MGS distribution can be yielded as

$$
\begin{aligned}
\mathcal{R} & =-\frac{1}{A} \log _{2}\left\{\sum_{j=1}^{K} \frac{\sigma_{j}\left(m_{s}\right)_{\beta_{j}} B\left(\beta_{j}, m_{s}+A\right)}{\left(m_{s}-1\right)^{\beta_{j}}}\right. \\
& \left.\times{ }_{2} F_{1}\left(\beta_{j}+m_{s}, \beta_{j} ; \beta_{j}+m_{s}+A ; 1-\frac{\zeta_{j}}{m_{s}-1}\right)\right\}
\end{aligned}
$$

Plugging (5) in (29) and recalling [15, eq. (3.194.3)], the asymptotic of the EC at $\bar{\gamma} \rightarrow \infty, R^{\infty}$, can be deduced as

$$
R^{\infty} \simeq-\frac{1}{A} \log _{2}\left\{\sum_{j=1}^{K} \frac{\sigma_{j}\left(m_{s}\right)_{\beta_{j}}}{\left(m_{s}-1\right)^{\beta_{j}}} B\left(\beta_{j}, A-\beta_{j}\right)\right\}
$$

\section{E. Average AUC of Energy Detection}

The average AUC, $\overline{\mathcal{A}}$, is a single figure of merit of ED that can be computed by [20, eq. (20)/ eq. (21)]

$$
\overline{\mathcal{A}}=1-\sum_{l=0}^{u-1} \sum_{i=0}^{l} \frac{\left(\begin{array}{c}
l+u-1 \\
l-i
\end{array}\right)}{2^{l+i+u} i !} \int_{0}^{\infty} \gamma^{i} e^{-\frac{\gamma}{2}} f_{\gamma}(\gamma) d \gamma
$$

where $\left(\begin{array}{l}a \\ b\end{array}\right)=\frac{a !}{b !(a-b) !}$ is the binomial coefficient.

Substituting (6) into (32) and utilizing [16, eq. (07.33.07.0001.01)], $\overline{\mathcal{A}}$ can be obtained as in (33) shown at the top of this page.

The average AUC at high $\bar{\gamma}$ value, $\overline{\mathcal{A}}^{\infty}$, can be evaluated after inserting (5) in (32) and invoking [15, eq. (3.381.4)] as

$$
\overline{\mathcal{A}}^{\infty} \simeq 1-\sum_{l=0}^{u-1} \sum_{i=0}^{l} \sum_{j=1}^{K} \frac{\sigma_{j}\left(m_{s}\right)_{\beta_{j}}\left(\begin{array}{c}
l+u-1 \\
l-i
\end{array}\right) \Gamma\left(\beta_{j}+i\right)}{2^{l+u-\beta_{j}}\left(m_{s}-1\right)^{\beta_{j}} i !}
$$

\section{Complexity Analysis}

A comparison between the complexity of the OP and EC of this work and the exact expressions is shown in Table I. In this table, $\Psi(k)=M(k \log k) \log \log k, k$ is the number of digits, $M$ refers to the complexity of the multiplication algorithm and HT denotes the higher transcendental functions (e.g., Gamma, Beta, Meijer's $G$, and hypergeometric) [21].

From Table I, one can see that (6) and (30) have less number of HT functions, as well as numerical operations than $[9$, eq.
TABLE I

COMPLEXITY COMPARISON OF THE OP AND EC WITH EXACT FORMATS

\begin{tabular}{|c|c|c|c|c|c|}
\hline \multirow{2}{*}{$\begin{array}{c}\text { Function/ } \\
\text { Operation }\end{array}$} & \multicolumn{4}{|c|}{ Number of Functions or Operations } & \multirow{2}{*}{ Complexity } \\
\cline { 2 - 5 } & {$[9$, Eq. (25)] } & Eq. (6) & {$[19$, Eq. (8)] } & Eq. (30) & \\
\hline HT & $4 K+6$ & $4 K+2$ & $8 K+10$ & $5 K+2$ & $\mathcal{O}\left(M(k) \log ^{2} k\right)$ \\
\hline Power & $K+3$ & $K+2$ & $K+2$ & $K+2$ & $\mathcal{O}(M(k) \log k)$ \\
\hline Division & $K+1$ & $K$ & $K+1$ & $K+1$ & $\mathcal{O}(M(k))$ \\
\hline Multiplication & $5 K+7$ & $5 K+2$ & $8 K+10$ & $5 K+3$ & $\mathcal{O}(M(k))$ \\
\hline Sum & $K+1$ & $K$ & $K$ & $K-1$ & $\mathcal{O}(k)$ \\
\hline Factorial & $K+1$ & $K$ & $K+1$ & $K$ & $\mathcal{O}(\Psi(k))$ \\
\hline
\end{tabular}

(25)] and [19, eq. (8)], respectively. Hence, in addition to their deriving in closed-form, our expressions mathematically simpler. Furthermore, the difference in the computational intricacy between the exact and MGS approaches becomes large when the statistics of the RVs, namely, sum, maximum, products, and ratio, are employed. This is due to increasing in the number of the complicated functions and operations.

\section{Analytical and Simulation Results}

In this section, the numerical, simulation, and asymptotic results are presented for different scenarios. To achieve MSE $\leq 10^{-5}$, we have chosen $K=15$.

Fig. 1 illustrates the OP versus normalized $\varphi$ for $\bar{\gamma}=5 \mathrm{~dB}$. Moreover, Figs. 2-3 explain the ABEP for BPSK modulation, the normalized ACC and EC for $A=3.5$, and the average complementary of AUC (CAUC), $1-\overline{\mathcal{A}}$, for $u=3$ versus $\bar{\gamma}$, respectively. Three cases for $m_{s}$ which are heavy $\left(m_{s}=1.5\right)$, moderate $\left(m_{s}=5.5\right)$, and light $\left(m_{s}=50\right)$ are studied.

From the provided results, it is clear that the performance becomes better when $\kappa$ and/or $\mu$ increase. This is because the high values of $\kappa$ and $\mu$ indicate that the total power of the scattered waves is less than that of the dominant components and large number of multipath clusters arrive at the receiver, respectively. Besides, the increasing in $m$ and/or $m_{s}$ means the impacts of the shadowing on the received signal are low. However, $m$ has higher effect on the performance than $m_{s}$ due to improving in the power of the dominant components.

In Fig. 3, one can see that the ACC is higher than the EC for the same scenario. This refers to the impact of the system delay on the EC whilst its ignored in the ACC. Moreover, the EC is related to the average AUC via $u$ [20]. This relationship explains how the low quality of the received signal by the unlicensed user would reduce the detectability of the ED.

For further validation, a comparison between our approximate results and the exact analysis is carried out in Figs. 1 and 3. As anticipated, a good matching between the results can be observed. But, a MGS approach has low mathematical complexity than [9] and [19]. Furthermore, a perfect agreement between the numerical results and their simulation counterparts as well as the asymptotic behaviour at high $\bar{\gamma}$ can be noted which proves the correctness of our expressions. 


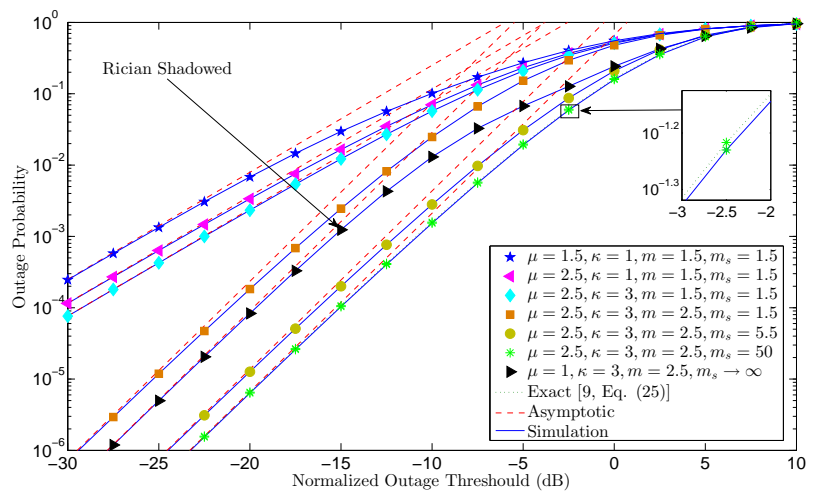

Fig. 1. OP versus normalized outage threshold for $\varphi=5 \mathrm{~dB}$.

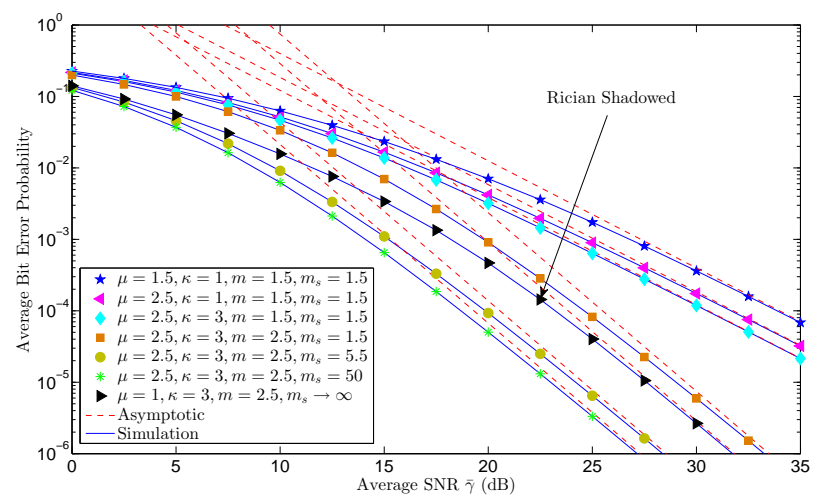

Fig. 2. ABEP for BPSK versus average SNR.

\section{CONCLUSIONS}

In this paper, a highly accurate approximate unified composite multipath/shadowed representation that is based on MG and inverse Nakagami- $m$ distributions was proposed. This model was then applied to the double shadowed $\kappa-\mu$ fading. The exact and asymptotic expressions of the OP, ABEP, ACC, EC and average AUC over MGS model were derived. The results for different values of the fading parameters were presented and compared with the exact models. The provided expressions can be reduced for many fading conditions, such as, $\kappa-\mu$ shadowed [5], $\kappa-\mu /$ inverse Gamma [6], and Fisher Senedcor $\mathcal{F}$ [17].

\section{REFERENCES}

[1] M. K. Simon and M.-S. Alouini, Digital Communications over Fading Channels. New York: Wiley, 2005.

[2] M. D. Yacoub, "The $\kappa-\mu$ distribution and the $\eta-\mu$ distribution," IEEE Antennas Propag. Mag., vol. 49, no. 1, pp. 68-81, Feb. 2007.

[3] J. Zhang et al., "Performance analysis of digital communication systems over composite $\eta$ - $\mu$ /gamma fading channels," IEEE Trans. Veh. Technol., vol. 61, no. 7, pp. 3114-3124, Sep. 2012.

[4] P. C. Sofotasios and S. Freear, "On the $\kappa-\mu /$ gamma composite distribution: A generalized multipath/shadowing fading model," in Proc. IEEE IMOC, Brazil, Oct. 2011, pp. 390-394.

[5] J. F. Paris, "Statistical characterization of $\kappa-\mu$ shadowed fading," IEEE Trans. Veh. Technol., vol. 63, no. 2, pp. 518-526, Feb. 2014.

[6] S. K. Yoo et al., "Effective capacity analysis over generalized composite fading channels" IEEE Access, vol. 8, pp. 123756-123764, Jul. 2020.

[7] N. Simmons, et al., "Double shadowing the Rician fading model," IEEE Wireless Commun. Lett., vol. 8, no. 2, pp. 344-347, April 2019.

[8] P.S. Bithas, V. Nikolaidis, A.G. Kanatas and G.K. Karagiannidis, "UAVto-Ground communications: Channel modeling and UAV selection," IEEE Trans. Veh. Technol., vol. 68, no. 8, pp. 5135-5144, Aug. 2020.

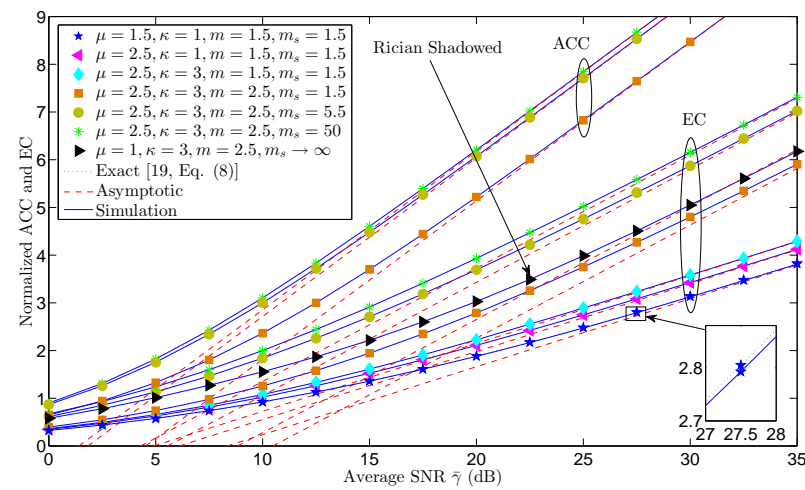

Fig. 3. Normalized ACC and EC with $A=3.5$ versus average SNR.

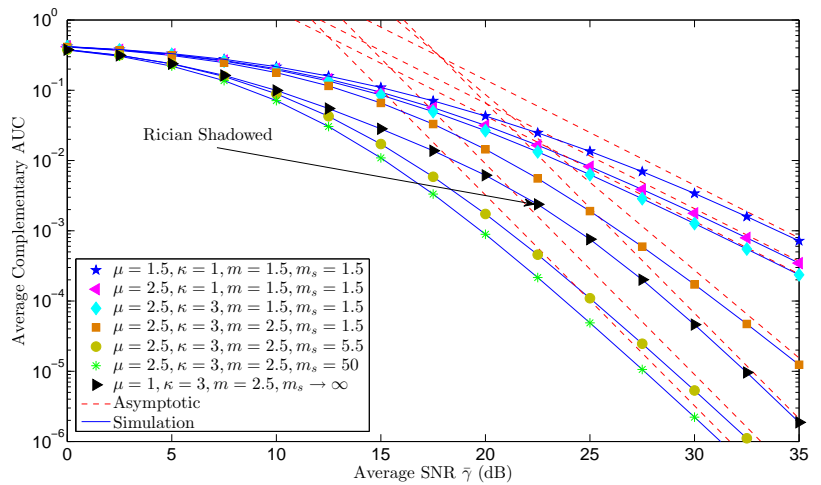

Fig. 4. Complementary AUC for $u=3$ versus average SNR.

[9] N. Simmons, et al., "On shadowing the $\kappa-\mu$ fading model," IEEE Access, vol. 8, pp. 120513-120536, Jun. 2020.

[10] S. Atapattu, C. Tellambura, and H. Jiang, "A mixture gamma distribution to model the SNR of wireless channels," IEEE Trans. Wireless Commun., vol. 10, no. 12, pp. 4193-4203, Dec. 2011.

[11] H. Al-Hmood and H. S. Al-Raweshidy, "Unified modeling of composite $\kappa-\mu /$ gamma, $\eta$ - $\mu /$ gamma, and $\alpha-\mu /$ gamma fading channels using a mixture gamma distribution with applications to energy detection," IEEE Antennas Wireless Propag. Lett., vol. 16, pp. 104-108, Apr. 2017.

[12] H. Al-Hmood and H. S. Al-Raweshidy, "Unified approaches based effective capacity analysis over composite $\alpha-\eta-\mu /$ gamma fading channels," Electronics Lett., vol. 54, no. 13, pp. 852-853, Jun. 2018.

[13] L. Kong and G. Kaddoum, "Secrecy characteristics with assistance of mixture gamma distribution," IEEE Wireless Commun. Lett., vol. 8, no. 4, pp. 1086-1089, Aug. 2019.

[14] H. Al-Hmood and H. S. Al-Raweshidy, "Unified analysis of channel capacity under different adaptive transmission policies," Electronics Lett., vol. 56, no. 2, pp. 87-89, Jan. 2020.

[15] I. S. Gradshteyn, and I. M. Ryzhik, Table of Integrals, Series and Products, 7th edition. Academic Press Inc., 2007.

[16] Wolfram Research, Inc., 2020, [Online]. Available: http://functions.wolfram.com/id. Accessed on: Oct. 2020.

[17] S. K. Yoo et al., "A comprehensive analysis of the achievable channel capacity in $\mathcal{F}$ composite fading channels," IEEE Access, vol. 7, pp. 34078-34094, Feb. 2019

[18] A.M. Salhab and M.H. Samuh, , "Accurate performance analysis of reconfigurable intelligent surfaces over Rician fading channels," IEEE Wireless Commun. Lett., to appear, 2021.

[19] R. Singh and M. Rawat, "On the performance analysis of effective capacity of double shadowed $\kappa$ - $\mu$ fading channels," 2019 IEEE Region 10 Conference (TENCON), Kochi, India, 2019, pp. 806-810.

[20] H. Al-Hmood, and H. S. Al-Raweshidy, "On the effective rate and energy detection based spectrum sensing over $\alpha-\eta-\kappa-\mu$ fading channels," IEEE Trans. Veh. Technol., vol. 69, no. 8, pp. 9112-9116, Aug. 2020.

[21] H.S. Silva et al., "Outage probability of the product of two Beaulieu-Xie, $\eta-\mu, \kappa-\mu$, or $\alpha-\mu$ random variables," IEEE Antennas Wireless Propag. Lett., vol. 19, no. 12, pp. 2182-2186, Dec. 2020. 